\title{
Índice de vulnerabilidade socioambiental: uma proposta metodológica utilizando o caso do Rio de Janeiro, Brasil
}

\author{
Socio-environmental vulnerability index: a methodological \\ proposal based on the case of Rio de Janeiro, Brazil
}

Fernanda Siqueira Malta ${ }^{1}$

Eduarda Marques da Costa ${ }^{2}$

Alessandra Magrini ${ }^{3}$

${ }^{1}$ Diretoria de Pesquisas, Instituto Brasileiro de Geografia e Estatística. Av. Chile $500 / 8^{\circ}$, Centro. 20031-170 Rio de Janeiro RJ Brasil.fernanda.malta@ ibge.gov.br

${ }^{2}$ Instituto de Geografia e Ordenamento do Território, Universidade de Lisboa.

Lisboa Estremadura Portugal.

${ }^{3}$ Programa de Planejamento Energético e Ambiental, Universidade Federal do Rio de Janeiro. Rio de Janeiro RJ Brasil.

\begin{abstract}
The term vulnerability has been more frequently used in several studies, striving to better understand the specificities and needs of different population groups. The scope of this study was to identify, characterize and analyze populations in situations of socio-environmental vulnerability in Rio de Janeiro city, consolidating social, economic, environmental, health and public security indicators in a synthesis index - the Socio-Environmental Vulnerability Index. The data sources used were the IBGE-2010 Demographic Census, the Geo-Rio Foundation and the Public Security Institute of the state of Rio de Janeiro. The methodology integrated Multicriteria Decision Analysis into a Geographic Information System. According to our results, the socio-environmental vulnerability in Rio de Janeiro city is aggravated by risk situations and environmental degradation. Those aspects are accentuated by the population density in shantytown areas, where the most disadvantaged strata exist in a process of environmental and urban exclusion. The study makes it possible to locate spatially vulnerable areas, emphasizing the importance of these tools to guide resource allocation, formulation and implementation of more effective public policies.
\end{abstract}

Key words Vulnerability analysis, Vulnerable populations, Geographic information systems, Decision support techniques
Resumo O termo vulnerabilidade tem sido utilizado com mais frequência, visando uma melhor compreensão acerca dos diferentes grupos populacionais, suas especificidades e necessidades. $O$ objetivo deste estudo foi identificar, caracterizar e analisar populações em situação de vulnerabilidade socioambiental no município do Rio de Janeiro, consolidando indicadores sociais, econômicos, ambientais, de saúde e de segurança pública, em um índice de sintese - o Índice de Vulnerabilidade Socioambiental. A metodologia foi baseada em análise multicritério de apoio à decisão integrada a um Sistema de Informação Geográfica. As fontes de dados utilizadas foram o Censo Demográfico do IBGE-2010, Fundação Geo-Rio e Instituto de Segurança Pública do estado do Rio de Janeiro. Os resultados demonstram que a vulnerabilidade socioambiental no Rio de Janeiro é agravada por situações de risco e degradação ambiental. Estes aspectos são acentuados pelo adensamento das áreas faveladas, onde os estratos mais vulneráveis residem, em um processo de exclusão ambiental e urbana. O estudo permite localizar espacialmente áreas mais vulneráveis, enfatizando a importância destas ferramentas para guiar alocação de recursos, formulação e implementação de políticas públicas mais adequadas.

Palavras-chave Análise de vulnerabilidade, Populações vulneráveis, Sistemas de informação geográfica, Técnicas de apoio para a decisão 


\section{Introdução}

Nos últimos anos, o termo vulnerabilidade tornou-se palavra-chave nos estudos sobre risco ambiental e mudanças climáticas. Ao mesmo tempo, e esta pode ser uma das razões para a sua crescente popularidade, a vulnerabilidade é um termo bastante difuso ${ }^{1}$. Diversas definições sobre vulnerabilidade emergiram e são utilizadas em diferentes contextos disciplinares, quer relacionada a sustentabilidade ${ }^{2}$, aos riscos naturais e ambientais ${ }^{3,4}$, no contexto das mudanças climáticas $^{5,6}$, quer nas áreas sociais ${ }^{7}$ e econômicas ${ }^{8}$. Outra abordagem da vulnerabilidade de grande relevância é a verificada no contexto da saúde ${ }^{9,10}$. O conceito de vulnerabilidade é bem amplo, sendo desta forma adaptado para cada área de conhecimento ${ }^{11}$.

Esse estudo propõe a elaboração de um índice para analisar o construto "vulnerabilidade socioambiental”, integrando processos sociais, econômicos e de infraestrutura urbana relacionados à precariedade das condições de vida da população (trabalho, educação, renda, saneamento, mobilidade) com as condições ambientais, de saúde e de segurança pública.

A construção do índice se sustenta na produção de indicadores. Estes podem ser definidos como uma medida, na maioria das vezes quantitativa, utilizada para substituir, quantificar ou operacionalizar um conceito abstrato ${ }^{12}$. São ferramentas bastante eficazes para subsidiar atividades de planejamento e formulação de políticas, alocação de recursos e definições de prioridades nas diferentes esferas de governo. Indicadores permitem por exemplo, o monitoramento das condições de vida de uma população e também da conjuntura econômica de um país ${ }^{13,14}$. Um bom indicador deve ser sensível à mudança de condições do ambiente e da sociedade, ser específico em relação ao problema analisado, ser reprodutível segundo padrões metodológicos estabelecidos, proporcionar uma pronta resposta, ser entendido pela população leiga, ser robusto para mudanças de metodologia e estar disponível ou ser de baixo custo ${ }^{15}$.

A construção de indicadores para avaliar as condições de vida e monitorar políticas públicas ganhou força na década de 1990, com a apresentação pelas Nações Unidas do índice de Desenvolvimento Humano (IDH), idealizado pelo economista Mahbub ul Haq, com a colaboração do economista Amartya Sen, ganhador do Prê- mio Nobel de Economia de $1998^{16}$. O IDH é uma medida resumida do progresso a longo prazo em três dimensões básicas do desenvolvimento humano: renda, educação e saúde. Este índice obteve grande repercussão mundial devido principalmente à sua simplicidade, fácil compreensão e pela forma mais holística e abrangente de mensurar o desenvolvimento. Apesar de o IDH ampliar a perspectiva sobre o desenvolvimento humano, ele não abrange nem esgota todos os aspectos de desenvolvimento, dentre eles a questão da vulnerabilidade. Desta forma, novos índices foram desenvolvidos para mensurar uma diversidade de temas não abrangidos pelo IDH.

No Brasil, nas décadas de 1990 e 2000, diversas ações para a elaboração de índices que pudessem retratar a realidade socioeconômica de diferentes grupos populacionais foram produzidas. Entre elas destacam-se o Índice de Exclusão/ Inclusão Social ${ }^{17}$, Índice de Desenvolvimento da Família-IDF ${ }^{18}$, Índice de Vulnerabilidade Juvenil - IVJ $^{19}$ e Índice de Qualidade de Vida Urbana-IQVU ${ }^{20}$.

A partir de 2010, outros índices foram desenvolvidos para embasar o desenvolvimento de políticas públicas especificamente voltadas para grupos populacionais considerados mais vulneráveis. Alguns exemplos são o Índice Paulista de Vulnerabilidade Social da Fundação Seade de São Paulo, o Índice de Vulnerabilidade da Saúde da Prefeitura Municipal de Belo Horizonte, o Índice de Vulnerabilidade Social do Instituto de Pesquisas Econômicas Aplicadas e o Índice de Vulnerabilidade Municipal da Fundação Oswaldo Cruz.

A questão da vulnerabilidade é complexa e cada situação, população vulnerável e região possui a necessidade de uma informação específica, e por esse motivo existem diversos índices, cada um desenvolvido para uma determinada realidade, com objetivos e utilizações diversas.

Diante deste contexto, o objetivo do presente estudo consiste em identificar, caracterizar e analisar as populações em situação de vulnerabilidade socioambiental, por meio da construção de um índice representativo de variáveis sociais, econômicas, de infraestrutura urbana, ambientais, de saúde e de segurança pública. Contribuindo, desta forma, com subsídios para suporte à formulação e implantação de políticas públicas, pois para estas ações é fundamental localizar espacialmente as áreas que concentram os segmentos populacionais mais vulneráveis nas dimensões consideradas. 


\section{Vulnerabilidade socioambiental no Rio de Janeiro}

Além da maior concentração populacional do estado, o município do Rio de Janeiro é uma região reconhecida de longa data pela sua desigualdade social, além dos problemas de infraestrutura urbana, riscos ambientais, carências no sistema de saúde e na segurança pública ${ }^{21-23}$. Esta realidade justifica o estudo da vulnerabilidade socioambiental no município, objetivando guiar a elaboração de políticas públicas e alocação de recursos públicos mais adequados e baseados em evidências científicas, resultante de um diagnóstico realizado com informação adequada ao território, escala e período de tempo.

O Rio de Janeiro é a cidade brasileira com os maiores contingentes de pessoas vivendo em aglomerados subnormais, nome técnico utilizado pelo Instituto Brasileiro de Geografia e Estatística (IBGE) para designar locais como habitações informais, construídas com materiais frágeis, invasões e comunidades com, no mínimo, 51 domicílios. Outro critério-chave para classificar essas áreas como aglomerados subnormais é a carência ou a inadequação de serviços públicos básicos, como por exemplo, abastecimento de água, esgotamento sanitário e serviço de coleta de lixo, além de, em geral, serem locais dispostos de forma densa e desordenada.
Segundo o Censo Demográfico de 2010, 23,0\% da população do Rio de Janeiro vive em aglomerados subnormais, mais popularmente denominados de favelas. A proporção de pessoas vivendo nesses locais varia significativamente no município, com amplo predomínio na região Central, na qual, embora em termos absolutos, a população residente em favelas seja a menor do município, sua proporção em relação à população total é a maior, representando 35\% dos habitantes da região. Em seguida, aparece a região Norte do município com $27 \%$ da sua população vivendo em favelas, depois região da Barra da Tijuca e Jacarepaguá com 26\%, zona sul com 17\% e, por último, a Zona Oeste com 16\%. A Figura 1 mostra o espalhamento dos 763 aglomerados subnormais no município do Rio de Janeiro.

A existência desta quantidade de aglomerados subnormais aponta para um grave problema relacionado à incongruência entre políticas habitacionais do município e à demanda por moradias. O crescimento da população nessas regiões, entre 2000 e 2010, foi de 27,65\%, enquanto a cidade regular, excetuando os moradores das favelas, cresceu a um ritmo oito vezes menor, apenas 3,4\%.

A presença dessas favelas torna-se um indicador trágico da dinâmica econômica, e revela o resultado da falta de políticas sociais eficazes ao longo das últimas décadas. Na cidade do Rio de Janeiro, centenas de favelas, a maioria delas em

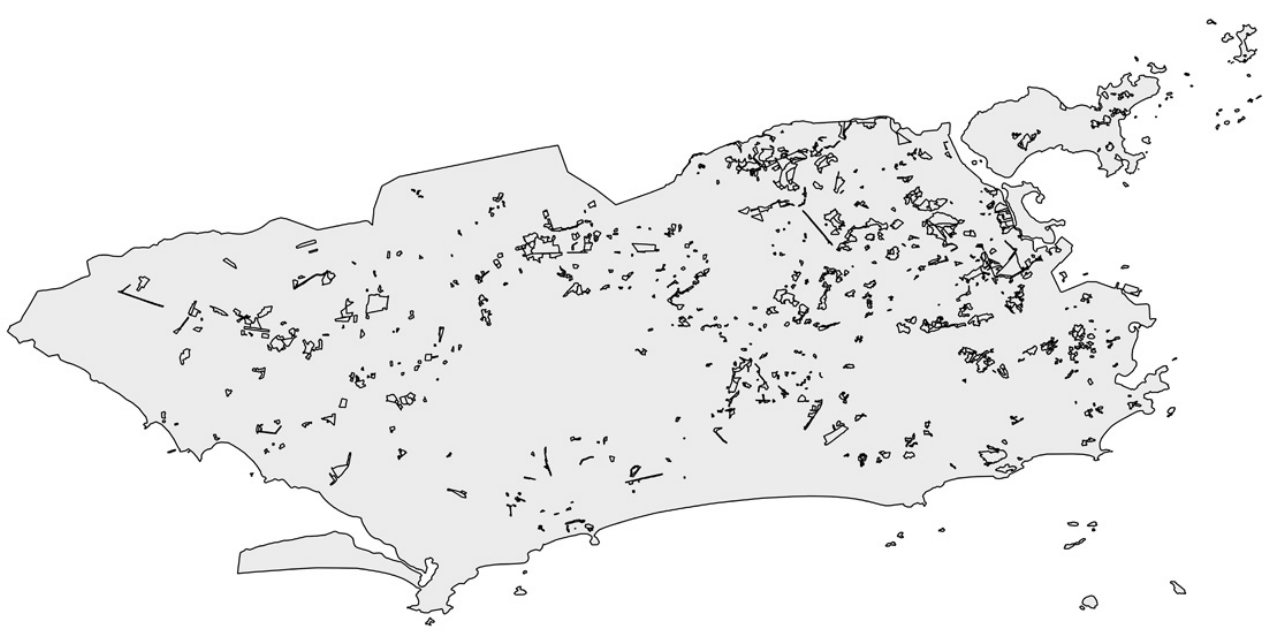

Aglomerados subnormais

Figura 1. Aglomerados subnormais no município do Rio de Janeiro em 2010. Fonte: IBGE, 2010. 
encostas, ou nas margens dos rios, criam condições de vida arriscadas, devido a deslizamentos de terra e inundações ${ }^{24}$.

A amplitude desses problemas urbanos e seus impactos em diferentes escalas territoriais fundamentam o interesse em compreender e analisar a questão das pessoas vivendo em situações de vulnerabilidade no Brasil e, em particular, no município do Rio de Janeiro, onde o problema das favelas se tornou crônico.

\section{Materiais e métodos}

O Índice de Vulnerabilidade Socioambiental (IVSA) foi construído através da integração da metodologia de análise multicritério de apoio à decisão (MCDA), mais especificamente do processo de hierarquia analítica (AHP), a um Sistema de Informação Geográfica (SIG) ${ }^{25}$.

O IVSA proposto é composto por quinze indicadores formulados com base em revisão bibliográfica e discussões com especialistas das áreas de Sociologia, Economia, Planejamento Urbano, Meio Ambiente, Saúde e Segurança.

Esses indicadores foram agrupados em três componentes: Socioeconômico, Infraestrutura Urbana e Ambiental, de Saúde e de Segurança. Cada componente é composto pelos seguintes indicadores.

Componente Socioeconômico:

Indicador 1: Percentual de mães chefes de família, sem ensino fundamental completo e com pelo menos um filho menor de 15 anos de idade (Fonte: Censo 2010);

Indicador 2: Percentual de crianças que vivem em domicílios em que nenhum dos moradores tem o ensino fundamental completo (Fonte: Censo 2010);

Indicador 3: Percentual de pessoas de 15 a 24 anos que não estudam, não trabalham e possuem renda domiciliar per capita igual ou inferior a meio salário mínimo (Fonte: Censo 2010);

Indicador 4: Proporção de pessoas com renda domiciliar per capita igual ou inferior a meio salário mínimo (Fonte: Censo 2010);

Indicador 5: Percentual de pessoas de 18 anos ou mais sem ensino fundamental completo e em ocupação informal (Fonte: Censo 2010).

Componente de Infraestrutura Urbana:

Indicador 6: Percentual de pessoas que vivem em domicílios com renda per capita inferior a meio salário mínimo e que gastam mais de uma hora até o trabalho (Fonte: Censo 2010);
Indicador 7: Razão de morador por domicílio (Fonte: Censo 2010);

Indicador 8: Percentual de domicílios sem bueiro/boca de lobo - abertura que dá acesso a caixas subterrâneas, por onde escoa a água proveniente de chuvas (Fonte: Censo 2010);

Indicador 9: Percentual de pessoas em domicílios com abastecimento de água e esgotamento sanitário inadequados (Fonte: Censo 2010);

Indicador 10: Percentual de pessoas em domićlios sem serviço de coleta de lixo (Fonte: Censo 2010).

Componente Ambiental, de Saúde e de Segurança:

Indicador 11: Susceptibilidade ao escorregamento (Fonte: Geo-Rio 2013) ${ }^{26}$;

Indicador 12: Risco de inundações e enchentes (Fonte: Índice de Susceptibilidade do Meio Físico à Inundação - 2010 ${ }^{27}$;

Indicador 13: Mortalidade até um ano de idade (Fonte: Censo 2010);

Indicador 14: Percentual de domicílios sem arborização no logradouro (Fonte: Censo 2010);

Indicador 15: Letalidade Violenta (Fonte: Instituto de Segurança Pública do Governo do Estado do Rio de Janeiro - 2013$)^{28}$.

Os indicadores utilizados na construção do IVSA procuram destacar diferentes situações indicativas de exclusão e vulnerabilidade no território brasileiro, em uma perspectiva que vai além da identificação da pobreza entendida, apenas como insuficiência de recursos monetários. As componentes do IVSA correspondem a conjuntos de ativos, recursos ou estruturas, cujo acesso, ausência ou insuficiência, indicam que o padrão de vida se encontra baixo, sugerindo, no limite, o não acesso e a não observância dos direitos sociais.

Após a seleção dos indicadores apresentados anteriormente, a metodologia proposta dividese em três etapas. A entrada de dados é uma das principais e requer cuidados especiais. Os indicadores utilizados na construção do IVSA foram padronizados, tornando-se adimensionais, variando entre 0 e 1 , sendo maior a vulnerabilidade quanto mais próxima de 1 . É necessário ressaltar que zero não representa ausência de vulnerabilidade, mas o menor valor relativo, e vice-versa para o valor 1 . Após esta etapa, os indicadores padronizados foram transferidos para um SIG e a eles foi aplicada a metodologia de análise multicritério para construção do IVSA.

Por opção metodológica e simplicidade analítica, foi utilizado o método de análise multicritério do processo de hierarquia analítica (Analy- 
tic Hierarchy Process-AHP), um dos principais métodos da escola americana, criado na década de 70 por Thomas L. Saaty ${ }^{29}$. Este método possui como fundamento a estrutura hierárquica de um problema complexo, adequado à análise proposta por nosso estudo, relacionada à vulnerabilidade socioambiental. Esta estrutura consiste na definição do objetivo global e decomposição do sistema em vários níveis de hierarquia, o que possibilita a visualização do sistema como um todo e também de suas componentes. Além disso, é possível, analisar as interações das componentes da decomposição e verificar os impactos que as mesmas exercem sobre o sistema.

O AHP permite fazer uma abordagem de tomada de decisão estruturada utilizando o julgamento de especialistas e inclui cinco etapas: (i) definição do problema, (ii) construção hierárquica e desenvolvimento do problema em fatores componentes relacionados aos objetivos do problema, (iii) construção da matriz de comparação, (iv) cálculo do vetor Eigen, número principal de Eigen, índice de consistência e razão de consistência, e (v) se houver inconsistências no processo de decisão, revisão da matriz de comparação até chegar a um consenso. O fluxograma conceitual do AHP é apresentado na Figura 2.

A partir da construção da hierarquia, os critérios, ou seja, os 15 indicadores das três componentes do IVSA (Figura 3) foram comparados par-a-par, de acordo com sua importância para atingir o objetivo principal, a minimização da Vulnerabilidade Socioambiental. Esta comparação foi fundamentada na escala de relativa importância proposta por Saaty ${ }^{29}$, baseado na análise de especialistas das áreas de Sociologia, Economia, Planejamento Urbano, Meio Ambiente, Saúde e Segurança.

Após a normalização da matriz de comparação original foi possível calcular o peso de cada indicador (vetor de Eigen) e o número principal de Eigen $\left(\lambda_{\max }\right)$, que é obtido através do somatório do produto de cada elemento do vetor de Eigen pelo total da respectiva coluna da matriz comparativa original.

Com a obtenção de $\lambda_{\text {max }}$ foi possível calcular o valor do Índice de Consistência (IC) da matriz de comparação através da fórmula:

$$
\mathrm{IC}=\frac{\left(\lambda_{\max }-\mathrm{n}\right)}{(\mathrm{n}-1)},
$$

onde $n$ é a ordem da matriz, que para este caso é igual a 15.

Saaty ${ }^{29}$ propõe o cálculo da Razão de Consistência (RC), obtida pela fórmula:

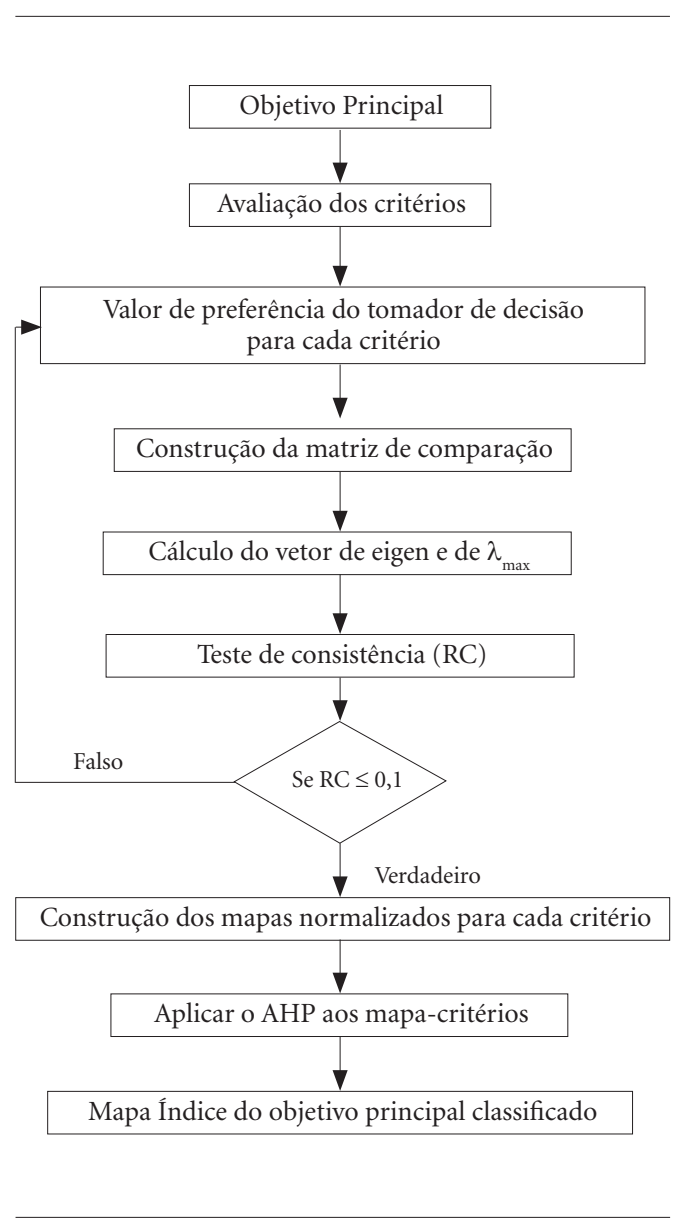

Figura 2. Fluxograma conceitual do AHP.

Fonte: Elaboração própria.

$$
\mathrm{RC}=\frac{\mathrm{IC}}{\mathrm{IR}},
$$

onde IC corresponde ao Índice de Consistência, e IR corresponde ao Índice de Consistência Aleatório, calculado para matrizes quadradas de ordem $n$ pelo Laboratório Nacional de Oak Ridge, nos Estados Unidos. Se RC for maior que 0,1, a matriz de comparação é inconsistente e deve ser revisada. Para $\mathrm{n}=15, \mathrm{IR}=1,59$ e obtemos o valor de $\mathrm{RC}=0,0997$. Como $\mathrm{RC}<0,1$ a matriz de comparação é consistente.

Após esta etapa é possível construir os mapas normalizados para cada indicador-critério, aplicar o AHP aos mapas-critérios e, por fim, obter o mapa índice do objetivo principal classificado.

O processamento estatístico dos dados foi feito através do software SAS, e o mapeamento, análises espaciais e AHP através do programa de código aberto QGIS. 


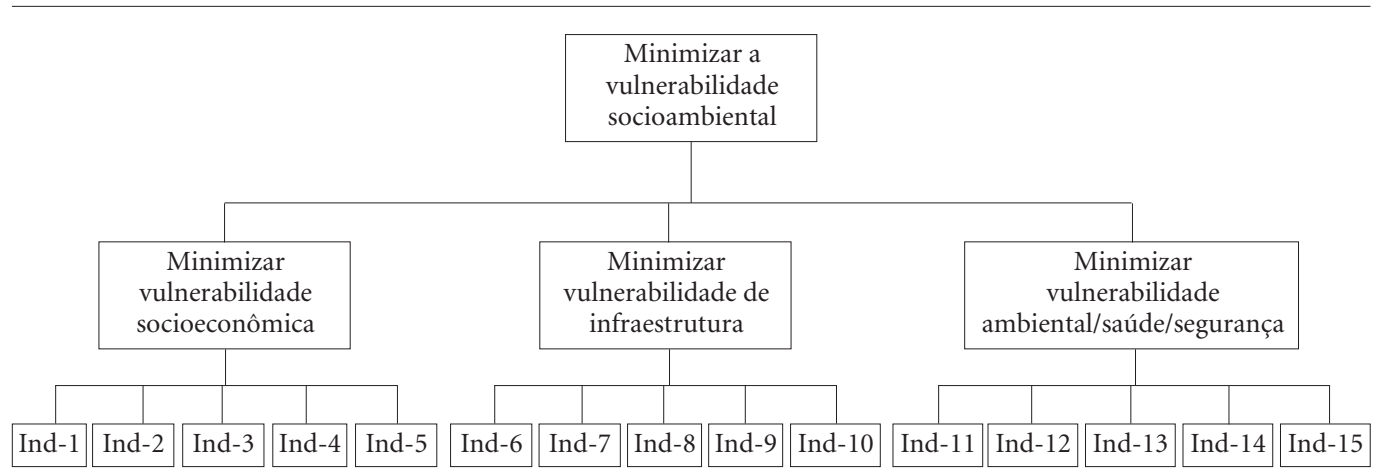

Ind-1: Minimizar o percentual de mães chefes de família, sem ensino fundamental completo e com pelo menos um filho menor de 15 anos de idade;

Ind-2: Minimizar o percentual de crianças que vivem em domićlios em que nenhum dos moradores tem o ensino fundamental completo;

Ind-3: Minimizar o percentual de pessoas de 15 a 24 anos que não estudam, não trabalham e possuem renda domiciliar per capita igual ou inferior a meio salário mínimo;

Ind-4: Minimizar a proporção de pessoas com renda domiciliar per capita igual ou inferior a meio salário mínimo;

Ind-5: Minimizar o percentual de pessoas de 18 anos ou mais sem ensino fundamental completo e em ocupação informal;

Ind-6: Minimizar o percentual de pessoas que vivem em domićlios com renda per capita inferior a meio salário mínimo e que gastam mais de uma hora até o trabalho;

Ind-7: Minimizar a razão de morador por domicílio;

Ind-8: Minimizar o percentual de domicílios sem bueiro/boca de lobo;

Ind-9: Minimizar o percentual de pessoas em domićlios com abastecimento de água e esgotamento sanitário inadequados;

Ind-10: Minimizar o percentual de pessoas em domicílios sem serviço de coleta de lixo;

Ind-11: Minimizar a suscetibilidade ao deslizamento;

Ind-12: Minimizar o risco de inundações e enchentes;

Ind-13: Minimizar a mortalidade até um ano de idade;

Ind-14: Minimizar o percentual de domicílios sem arborização no logradouro;

Ind-15: Minimizar a letalidade violenta.

Figura 3. Hierarquia de objetivos da análise multicritério.

Fonte: Elaboração própria.

\section{Área de Estudo}

A área de estudo abrange todo o município do Rio de Janeiro, capital do estado do Rio de Janeiro, localizado na região sudeste do Brasil. O Rio de Janeiro possui uma área de $1.200 \mathrm{~km}^{2}$, onde vivem aproximadamente 6,3 milhões de pessoas, segundo o Censo 2010, $40 \%$ de toda a população do estado. O município do Rio de Janeiro é uma região totalmente urbanizada e está dividido em Áreas de Planejamento (APs), uma divisão utilizada pela prefeitura para atender administrativamente todo o município.

A área de planejamento 1 (AP 1) é a região do centro histórico da cidade, e também a área que mais sofreu transformações do cenário urbano. A área de planejamento 2 (AP 2), conhecida como Zona Sul, corresponde à área de expansão da cidade, promovida por implantação do sistema de bondes, na segunda metade do século XIX, e está localizada entre o Oceano Atlântico e o Maciço da Tijuca. A área de planejamento 3 (AP 3), conhecida também como Zona Norte, concentra o maior contingente populacional do município (40\%), sendo a mais densamente povoada. A área de planejamento 4 (AP 4) possui uma extensa área de baixada, limitada pelos maciços da Tijuca, da Pedra Branca e pelo Oceano Atlântico. Esta região engloba os bairros da Barra da Tijuca e Jacarepaguá. A área de planejamento 5 (AP 5), também conhecida como Zona Oeste, apresenta a segunda maior população do município e a menor densidade demográfica. A baixa densidade ocorre por ser uma região de área territorial muito extensa. Nesta região localizam-se os três bairros mais populosos da cidade: Campo Grande, Bangu e Santa Cruz (Figura 4). 


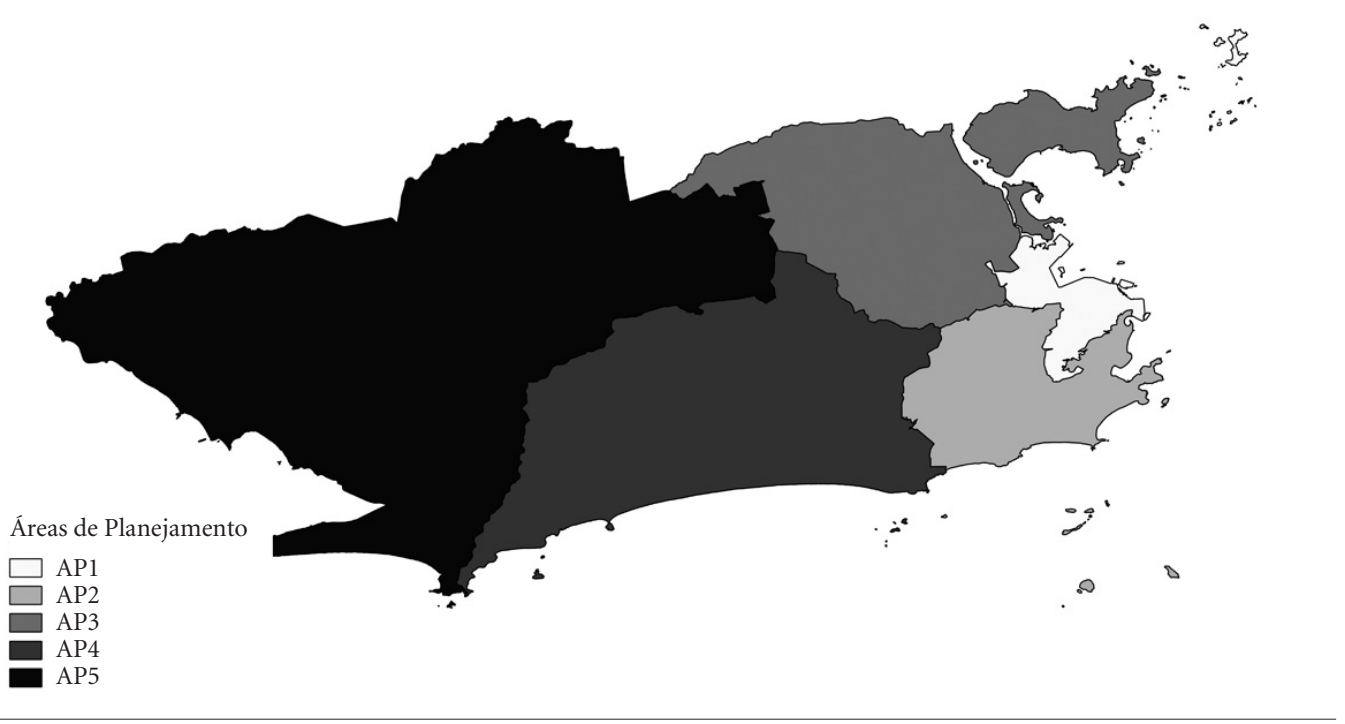

Figura 4. Município do Rio de Janeiro por Área de Planejamento. Fonte: Elaboração própria a partir de prefeitura do Rio de Janeiro, 2010.

\section{Resultados}

Como mencionado anteriormente, o IVSA varia entre 0 e 1 , sendo que quanto mais próximo a 1 , maior é a vulnerabilidade socioambiental, e inversamente, quanto mais próximo de 0 , menor. Para melhor aplicabilidade, este índice foi dividido em cinco faixas iguais:

0,000 a 0,200-Muito baixa vulnerabilidade;

0,201 a 0,400 -Baixa vulnerabilidade;

0,401 a 0,600-Média vulnerabilidade;

0,601 a 0,800 -Alta vulnerabilidade;

0,801 a 1,000-Muito alta vulnerabilidade.

Os resultados foram apresentados por APs, em conformidade com a abordagem territorial do macroplanejamento existente na prefeitura do Rio de Janeiro.

Aproximadamente, metade da área da AP 1 está classificada como média vulnerabilidade socioambiental $(48,3 \%), 27,4 \%$ como baixa vulnerabilidade socioambiental, 23,6\% como alta vulnerabilidade socioambiental, e $0,7 \%$ como vulnerabilidade socioambiental muito alta. O destaque da região de vulnerabilidade muito alta fica para a favela do Morro da Providência, que possui mais da metade dos indicadores que compõem o IVSA classificados como vulnerabilidade alta ou muito alta. Não há casos de muito baixa vulnerabilidade socioambiental nesta área de planejamento. A população desta região tem sido alvo de diversos estudos acerca de sua vulnerabilidade social acrescida ${ }^{30}$.
A AP 2, possui comportamento bem diferente da AP 1. 71,2\% da região está classificada como baixa vulnerabilidade socioambiental, $21,0 \%$ como média vulnerabilidade e 7,8\% como alta vulnerabilidade. Não há casos de muito baixa ou muito alta vulnerabilidade socioambiental. O destaque da região de alta vulnerabilidade fica para a favela da Rocinha, onde residem 69.161 habitantes, segundo o Censo 2010, sendo a maior favela do Brasil. A Rocinha apresenta indicadores altos ou muito altos da componente socioeconômica, baixos de infraestrutura urbana, muito altos de suscetibilidade ao escorregamento e altos de mortalidade infantil. Apesar de terem ocorrido investimentos em infraestrutura, como abastecimento de água, esgotamento e coleta de lixo, outros problemas socioeconômicos, de saúde e risco ambiental ainda estão muito presentes nesta região.

A AP 3 possui mais da metade da sua região caracterizada como média vulnerabilidade socioambiental (59,8\%), 20,7\% como baixa vulnerabilidade, $18,0 \%$ como alta e $1,5 \%$ como muito alta vulnerabilidade socioambiental. $\mathrm{Na}$ região classificada como muito alta vulnerabilidade destacam-se as favelas Fazenda Botafogo e Bairro da Pedreira. Nas duas regiões todos os indicadores socioeconômicos, além do indicador de mortalidade infantil, são classificados como muito altos.

A AP 4 encontra-se dividida em baixa vulnerabilidade $(43,3 \%)$ e média vulnerabilidade socioambiental $(43,5 \%)$. As áreas de alta vulnera- 
bilidade ocupam 13,2\% desta região. O destaque das áreas de alta vulnerabilidade fica para a favela do Rio das Pedras, onde segundo o Censo 2010, residem 54.793 habitantes, sendo a terceira maior do Brasil. Esta região possui praticamente todos os indicadores da componente socioeconômica classificados como alta ou muito alta vulnerabilidade, muito alto risco de inundação e enchente, além de muito alta letalidade violenta.

A região da AP 5 está dividida em áreas de média $(42,6 \%)$ e alta $(48,5 \%)$ vulnerabilidade socioambiental. Desta região apenas 2,0\% é classificada como baixa vulnerabilidade. A AP 5 possui o maior percentual de muito alta vulnerabilidade socioambiental em relação às outras áreas de planejamento $(6,9 \%)$, concentrado principalmente nos bairros de Guaratiba, Paciência e Santa Cruz, regiões estas que não são necessariamente áreas de favelas.

Ao analisarmos o resultado final do IVSA para todo o município do Rio de Janeiro, percebe-se que a maioria da região está classificada como média vulnerabilidade socioambiental (44,0\%). A seguir, 30,5\% do território está classificado como alta vulnerabilidade socioambiental, situação mais presente na AP 5. 21,9\% do território foi classificado como baixa vulnerabilidade socioambiental, situação predominante nas APs 1,2 e $4.3,6 \%$ foi classificado com muito alta vulnerabilidade socioambiental, com maior concentração na AP 5. Não ocorreram casos de muito baixa vulnerabilidade socioambiental (Figura 5).

\section{Discussão}

Segundo Malczewski e Ogryczak ${ }^{31}$, desde a década de 1990, tem ocorrido uso crescente da metodologia de análise multicritério no planejamento territorial e urbano. Atualmente, a análise multicritério vem sendo integrada aos SIGs, criando uma ferramenta robusta para apoiar processos de análise espacial através da modelagem e dar suporte e apoio a decisão em questões com distribuição e consequências espaciais. Os esforços para integrar o SIG e análise multicritério no final dos anos 80 e início dos anos 90 podem estar associados ao aumento do desenvolvimento dos SIGs ${ }^{25}$. Sharifi et al. ${ }^{32}$ afirmam ainda que a integração de SIG e análise multicritério fornecem uma metodologia importante no desenvolvimento de opções para reduzir os impactos ambientais e socioeconômicos, assim como para avaliação e solução destes impactos no território.

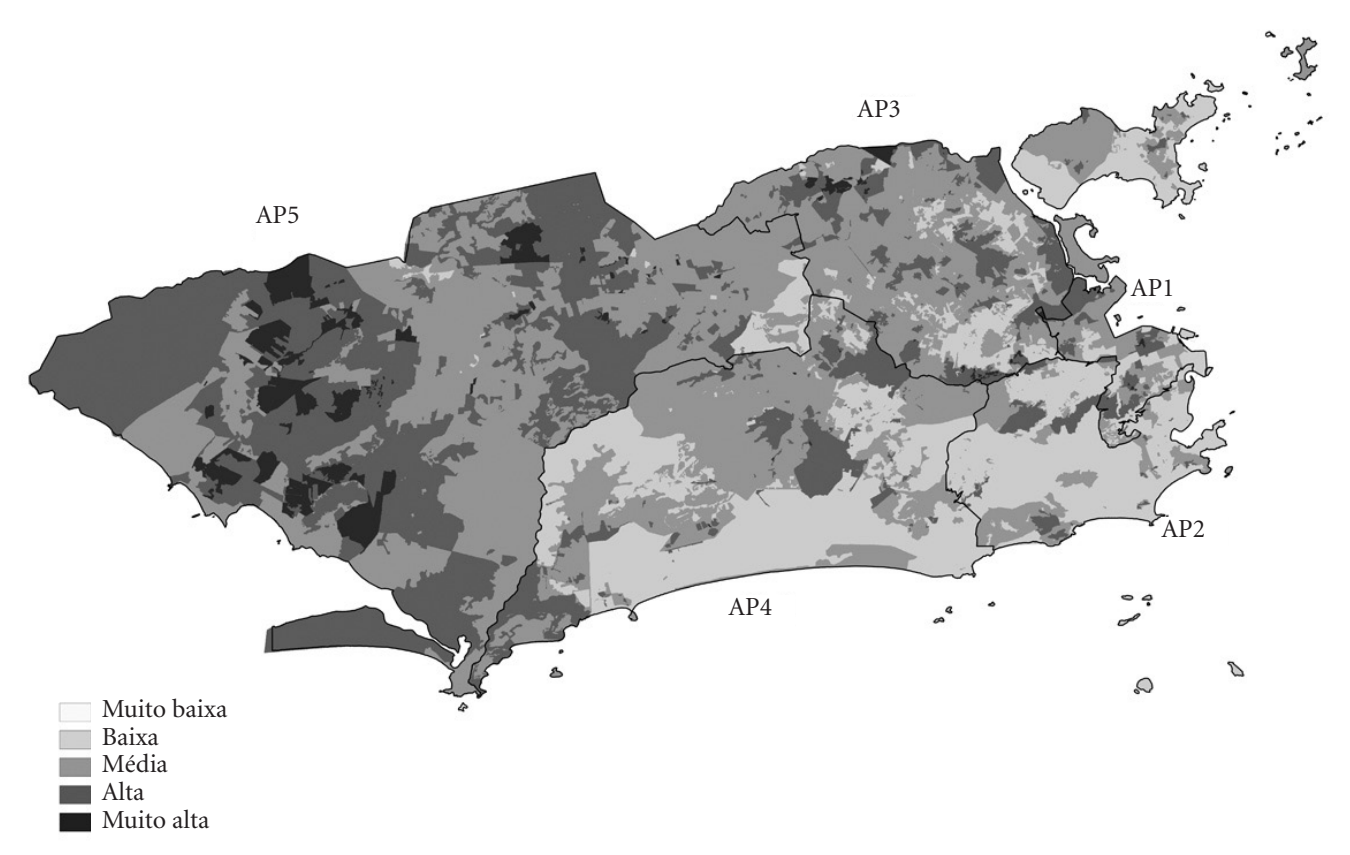

Figura 5. Mapa do Índice de Vulnerabilidade Socioambiental. 
A metodologia de integração entre SIG e análise multicritério utilizada no cálculo do IVSA corrobora os estudos de Malczewski ${ }^{33}$, onde o autor afirma que a integração entre as técnicas de tomada de decisão multicritérios e SIGs representa um avanço considerável nas análises espaciais envolvendo planejamento urbano, comparado às abordagens convencionais de sobreposição de mapas.

A importância desta metodologia pode ser verificada com o aumento de publicações nas mais diversas áreas e regiões do mundo. Como exemplos pode-se citar o estudo de Joerin et al., onde esta metodologia é utilizada no planejamento do uso da terra na Suíça ${ }^{34}$; Sener et al. ${ }^{35}$, na identificação de locais para construção de aterros sanitários na região do Lago Beys, na Turquia; Elsheikh et al. ${ }^{36}$, na construção de um mapa de risco de inundação em Terengganu, na Malásia; Wanyonyi et al. $^{37}$, na localização de potenciais sítios para ecoturismo no Quênia; Myagmartseren et $a .^{38}$, na identificação de regiões adequadas para o desenvolvimento urbano em Ulaanbaatar, Mongólia. No Brasil, Santos et al. ${ }^{39}$, na análise da fragilidade social da área urbanizada do município de Viçosa - MG; Oliveira et al. ${ }^{40}$, para determinar o Índice de Sustentabilidade de Expansão do Setor Sucroalcooleiro; e Gomes e Lins ${ }^{41}$, na análise da qualidade de vida urbana da população do município do Rio de Janeiro.

Desta forma, a metodologia de análise multicritério integrada ao SIG vem se consolidando como um recurso extremamente útil na gestão pública e privada. Para tanto é preciso que se entenda a ferramenta como recurso para reflexão das práticas e auxílio à tomada de decisão, garantindo a transparência e a possibilidade de incorporação de juízos de valor subjetivos no processo $^{42}$. No caso específico deste artigo, a integração das metodologias visa auxiliar os processos de planejamento urbano e de ordenação do território.

A vulnerabilidade socioambiental está diretamente relacionada à urbanização do Brasil, onde, em 2010, mais de $80 \%$ da população brasileira vivia em áreas urbanas. Além da urbanização concentrada, essa mudança ocorreu em apenas algumas décadas, de forma que as infraestruturas dessas cidades não acompanharam tal crescimento. Esse rápido e desordenado processo de urbanização trouxe uma série de consequências, em sua maioria negativas. A falta de planejamento urbano e de uma política econômica menos concentradora contribuiu para a ocorrência de alguns problemas, que persistem até hoje. Um dos principais problemas decorrente da acelerada urbanização brasileira foi a concentração da riqueza, e como consequência o aumento das desigualdades.

Essa formação desigual da estrutura social se expressa na estrutura urbana, ou seja, o direito à cidade não é justo e igual para todos os seus moradores. Desta forma, os grupos mais vulneráveis sofrem uma segregação socioespacial. No caso do município do Rio de Janeiro esta segregação ocorre nas regiões de favelas, confirmando as observações de Rodrigues ${ }^{43}$ em relação à luta pelo direito à cidade.

Através dos resultados encontrados neste artigo, percebe-se que os maiores valores para o IVSA se encontram em regiões de favelas, confirmando que é sobre as populações mais carentes que recai a maior parte dos efeitos negativos da urbanização, confirmando estudos iniciais de Cutter et al. ${ }^{44}$, onde os autores discutem a variação da vulnerabilidade no tempo e espaço, entre diferentes grupos sociais.

A luta pelo direito à cidade, e pelo direito à moradia, um de seus componentes centrais, emergiu como contraposição a um modelo de urbanização excludente, que ao longo de décadas de urbanização acelerada absorveu, em poucas e grandes cidades, grandes contingentes de pessoas mais carentes, sem jamais integrá-las efetivamente às cidades ${ }^{45}$. Além dos problemas socioeconômicos, uma das dimensões da luta pelo direito à cidade passa pelo direito a um ambiente saudável ${ }^{46,47}$, o que demanda o acesso a políticas de saneamento, habitação, segurança, infraestrutura e de saúde. A metodologia e os resultados obtidos para o IVSA podem servir como suporte para estas políticas, contribuindo na minimização da segregação socioespacial e, consequentemente, uma mudança no modelo de urbanização atual, de maneira que todos os moradores tenham o mesmo direito à cidade.

\section{Conclusão}

A metodologia de integração entre análise multicritério e SIG desenvolvida na construção do IVSA apresenta uma importante ferramenta para definir e validar políticas para grupos em situação de vulnerabilidade. A elaboração de um mapa-índice para o IVSA favorece a visualização de aspectos importantes dos processos de vulnerabilidade, sendo possível a desagregação em mapas das suas componentes e também mapas dos indicadores utilizados na sua construção. Desta 
forma é possível identificar áreas prioritárias carentes de políticas específicas e também favorecer o seu monitoramento.

A utilização de componentes socioeconômicas, de infraestrutura urbana, ambientais, de saúde e de segurança na construção do IVSA constitui uma combinação que representa bem a vulnerabilidade socioambiental e, no caso específico deste estudo a realidade dos grupos mais vulneráveis do município do Rio de Janeiro. Esses resultados devem ser levados em consideração pelo poder público e por outros organismos que lidam com esse contexto problemático no intuito de diminuírem as situações de vulnerabilidade e democratizarem o direito à cidade.

Para a sua aplicação em outras regiões, sugere-se a inclusão e/ou substituição de indicadores de acordo com a realidade da região a ser analisada. Desta forma, através do conhecimento espacial das áreas mais vulneráveis torna-se possível subsidiar a elaboração de planos de preparação e resposta para o enfrentamento destes problemas e, consequentemente, da sua mitigação.

\section{Colaboradores}

FS Malta trabalhou na concepção, delineamento do estudo, análise e interpretação dos dados, revisão de literatura, redação do artigo e aprovou a versão a ser publicada. EM Costa e A Magrini participaram do delineamento do estudo, orientação teórica, revisão final e aprovação da versão a ser publicada.

\section{Agradecimentos}

Este trabalho foi desenvolvido com o apoio do Conselho Nacional de Desenvolvimento Científico e Tecnológico, através da bolsa do Programa Ciências Sem Fronteiras para realização do Doutorado Sanduíche no Instituto de Geografia e Ordenamento do Território da Universidade de Lisboa. Além do financiamento do Instituto Brasileiro de Geografia e Estatística na forma de licença com vencimentos para realização de doutorado em período integral. 


\section{Referências}

1. Kuhlicke C, Scolobig A, Tapsell S, Steinfuhrer A, Marchi B. Contextualizing social vulnerability: findings from case studies across Europe. Natural Hazards 2011; 58(2):789-810.

2. Turner B II, Kasperson R, Matson P, McCarthy J, Corell R, Christensen L, Eckley N, Kasperson JX, Luers A, Martello ML, Polsky C, Pulsipher A, Schiller A. A framework for vulnerability analysis in sustainability science. Proc Natl Acad Sci USA 2003; 100(14):80748079.

3. Brasil. Ministério do Meio Ambiente (MMA). Vulnerabilidade Ambiental. Brasília: MMA; 2007.

4. Marandola Júnior E, Hogan DJ. Vulnerabilidades e riscos: entre Geografia e Demografia. Rev Bras Estud Popul 2005; 22(1):29-53.

5. Fussel HM, Klein R. Climate change vulnerability assessments: an evolution of conceptual thinking. Climatic Change 2006; 75(3):301-329.

6. Fundação Oswaldo Cruz (Fiocruz). Mapa da Vulnerabilidade da População dos Municípios do Estado do Rio de Janeiro Frente às Mudanças Climáticas. Rio de Janeiro: Fiocruz; 2014.

7. Instituto de Pesquisa Econômica Aplicada (IPEA). Atlas da Vulnerabilidade Social nos municípios brasileiros. Brasília: IPEA; 2015.

8. Fossati D. Economic vulnerability and economic voting in 14 OECD countries. Eur J Polit Res 2014; 53(1):116135.

9. Silva MAI, Mello FCM, Mello DF, Ferriani MGC, Sampaio JMC, Oliveira WA. Vulnerabilidade na saúde do adolescente: questões contemporâneas. Cien Saude Colet 2014; 19(2):619-627.

10. Eisenman DP, Wilhalme H, Tseng CH, Chester M, English P, Pincetl S, Fraser A, Vangalas S, Dhaliwal SK. Heat Death Associations with the built environment, social vulnerability and their interactions with rising temperature. Health Place 2016; 41(1):89-99.

11. Gallopín GC. Linkages between vulnerability, resilience, and adaptive capacity. Global Environmental Change 2006; 16(3):293-303

12. Jannuzzi PM. Indicadores sociais no Brasil: conceitos, fonte de dados e aplicações. Campinas: Alínea; 2012.

13. Carley M. Indicadores sociais: teoria e prática. Rio de Janeiro: Zahar; 1985.

14. United Nations (UN). Handbook on Social Indicators. New York: UN; 1989.

15. Barcellos C. Constituição de um sistema de indicadores socioambientais. In: Minayo MC, organizadora. Saúde e Ambiente Sustentável: Estreitando Nós. Rio de Janeiro: Fiocruz; 2002. p. 313-329.

16. United Nations. Human Development Report. Nova York: Oxford University Press; 1990.

17. Sposati A. Mapa da Exclusão. Inclusão Social da cidade de São Paulo. São Paulo: Educ; 1996.

18. Barros RP, Carvalho M, Franco S. O indice de desenvolvimento da família (IDF). Brasília: IPEA; 2003.

19. Fundação SEADE. Índice de Vulnerabilidade Juvenil (IVJ). São Paulo: Fundação SEADE; 2000.

20. Belo Horizonte. Secretaria Municipal de Planejamento, Orçamento e Informação (SMPOI). Série histórica IQVU 1994-2000-2006: notas metodológicas. Belo Horizonte: SMPOI; 2008.

21. Abreu MA. Evolução Urbana do Rio de Janeiro. Rio de Janeiro: IPLANRIO, Jorge Zahar Editor; 1987.
22. Carvalho JM. Os bestializados: o Rio de Janeiro e a República que não foi. São Paulo: Companhia das Letras; 1987.

23. Ferreira A. Favelas no Rio de Janeiro: nascimento, expansão, remoção e, agora, exclusão através de muros. Revista Bibliográfica de Geografía y Ciencias Sociales [periódico na Internet]. 2009; XIV(828): [cerca de 5 p.]. [acessado 2017 Mar 12]. Disponível em: http:// www.ub.es/geocrit/b3w-828.htm

24. Alves HPF, Torres HG. Vulnerabilidade socioambiental na cidade de São Paulo: uma análise de famílias e domicílios em situação de pobreza e risco ambiental. Revista São Paulo em Perspectiva 2006; 20(1):44-60.

25. Malczewski J. GIS-based multicriteria decision analysis: a survey of the literature. Int J Geogr Inf Sci 2006; 20(7):703-726.

26. Fundação Geo-Rio. Painel 2013: Estamos preparados para as chuvas? Rio de Janeiro: Fundação GEO-Rio; 2013.

27. Miranda FM. Índice de susceptibilidade do meio físico a inundações como ferramenta para o planejamento urbano [dissertação]. Rio de Janeiro: Universidade Federal do Rio de Janeiro; 2016.

28. Estado do Rio de Janeiro. Instituto de Segurança Pública (ISP-RJ). Taxa de Letalidade Violenta. Rio de Janeiro: ISP-RJ; 2013.

29. Saaty TL. The analytic hierarchy process: planning, priority setting, resource allocation. New York: McGraw -Hill International Book Co.; 1980.

30. Gutterres AS. O rumor e o terror na construção de territórios de vulnerabilidade na zona portuária do Rio de Janeiro. Mana 2016; 22(1):179-209.

31. Malczewski J, Ogryczak W. The multiple criteria location problem: 2. preference- based techniques and interactive decision support. Environ Plan A 1996; 28(1):69-98.

32. Sharifi MA, Toorn $W$ van den, Rico A, Emmanuel M. Application of GIS and multicriteria evaluation in locating sustainable boundary between the tunari $\mathrm{Na}-$ tional Park and Cochabamba City (Bolivia). J MultiCrit Decis Anal 2002; 11(1):151-164.

33. Malczewski J. GIS-based land-use suitability analysis: critical overview. Prog Plann 2004; 62(1):3-65

34. Joerin F, Thériault M, Musy A. Using GIS and outranking multicriteria analysis for land-use suitability assessment. Int J Geogr Inf Sci 2001; 15(2):153-174.

35. Sener S, Sener E, Nas B, Karagüzel R. Combining AHP with GIS for landfill site selection: A case study in the Lake Beysehir catchment area (Konya, Turkey). Waste Manag 2010; 30(1):2037-2046.

36. Elsheikh RFA, Ouerghi S, Elhag AR. Flood risk map based on GIS, and multi criteria techniques (case study Terengganu Malaysia). Journal of Geographic Information System 2015; 7(1):348-357.

37. Wanyonyi JW, Imwati A, Boitt M. GIS in analysis of potential sites for ecotourism - a case study of Kwale County. J Environ Sci Toxicol Food Technol 2016; 10(10):43-49.

38. Myagmartseren P, Buyandelger M, Brandt SA. Implications of a spatial multicriteria decision analysis for urban development in Ulaanbaatar, Mongolia. Mathematical Problems in Engineering [Internet]. $2017 \mathrm{Fev}$ [acessado 2017 Mar 12]. Disponível em: https://www. hindawi.com/journals/mpe/2017/2819795/cta/. 
39. Santos AP, Rocha SF, Abreu MVS, Calijuri ML, Santos PM. O uso da análise multicritério no mapeamento da fragilidade social da área urbanizada do município de Viçosa - MG. Revista Brasileira de Cartografia 2012; 64(5):635-643.

40. Oliveira DBB, Rodrigues JP, Silva LF, Oliveira PTS. Multi-criteria analysis in the strategic environmental assessment of the sugar and alcohol sector. Acta Scientiarum Technology 2012; 34(3):303-311.

41. Gomes EG, Lins MPE. Integrating Geographical Information Systems and multicriteria methods: a case study. Ann Oper Res 2002; 116(1):243-269.

42. Jannuzzi PM, Miranda WL, Silva DSG. Análise Multicritério e Tomada de Decisão em Políticas Públicas: Aspectos Metodológicos, Aplicativo Operacional e Aplicações. Informática Pública 2009; 11(1):69-87.

43. Rodrigues AM. Desigualdades socioespaciais - a luta pelo direito à cidade. Cidades 2007; 4(6):73-88.

44. Cutter SL, Boruff BJ, Shirley WL. Social vulnerability to environmental hazards. Soc Sci Quart 2003; 84(2):242261.

45. Rolnik R, Klink J. Crescimento econômico e desenvolvimento urbano: por que nossas cidades continuam tão precárias? Novos Estudos-CEBRAP 2011; 89(1):89109.

46. Cohen SC, Cynamon SE, Kligerman DC, Assumpção RF. Habitação saudável no Programa Saúde da Família (PSF): uma estratégia para as políticas públicas de saúde e ambiente. Cien Saude Colet 2004; 9(3):807-813.

47. Cohen SC, Bodstein R, Kligerman DC, Marcondes WB. Habitação saudável e ambientes favoráveis à saúde como estratégia de promoção da saúde. Cien Saude Colet 2007; 12(1):191-198.

Artigo apresentado em 08/08/2017

Aprovado em 04/09/2017

Versão final apresentada em 03/10/2017 